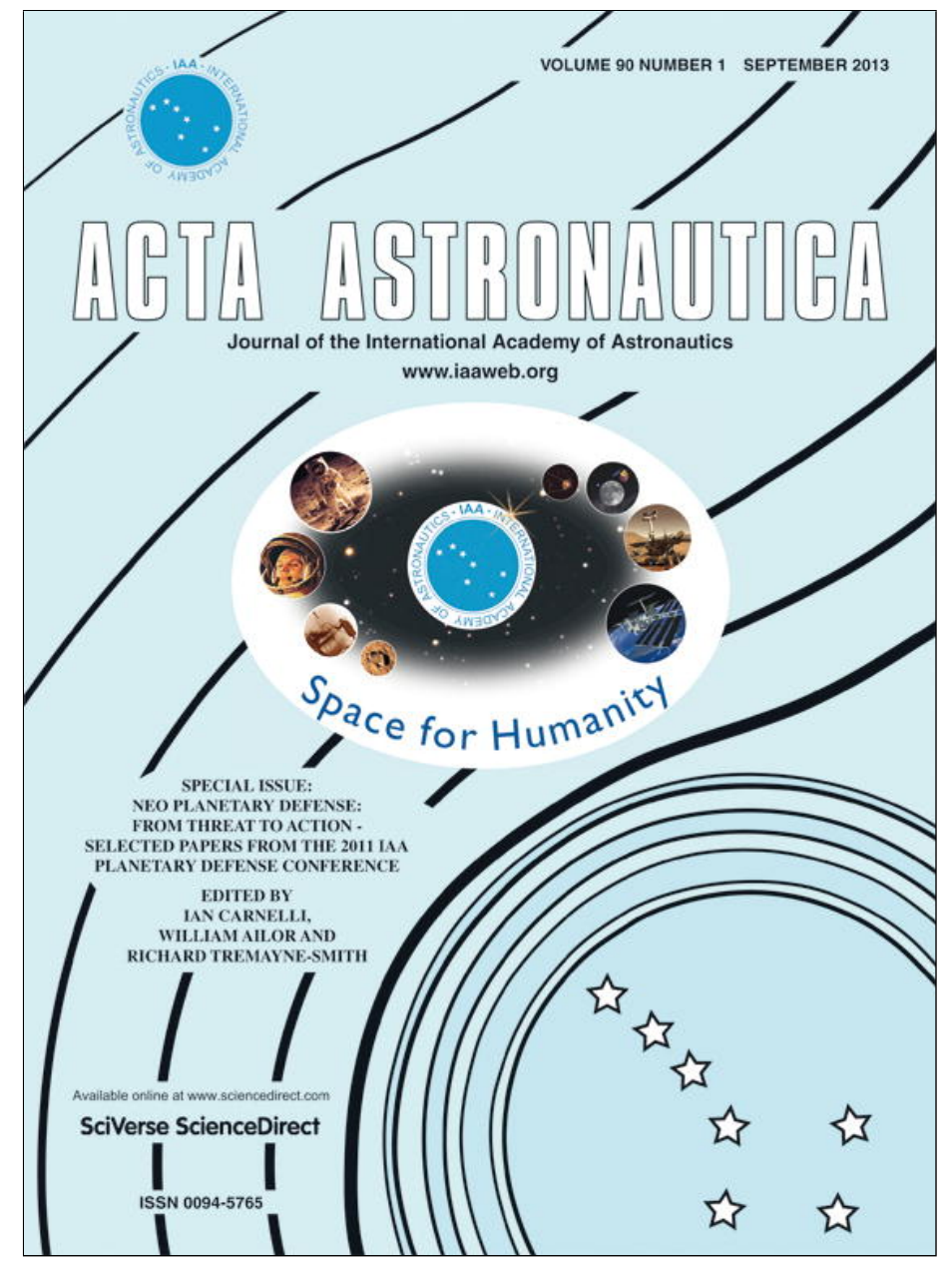

This article appeared in a journal published by Elsevier. The attached copy is furnished to the author for internal non-commercial research and education use, including for instruction at the authors institution and sharing with colleagues.

Other uses, including reproduction and distribution, or selling or licensing copies, or posting to personal, institutional or third party websites are prohibited.

In most cases authors are permitted to post their version of the article (e.g. in Word or Tex form) to their personal website or institutional repository. Authors requiring further information regarding Elsevier's archiving and manuscript policies are encouraged to visit: 


\title{
Probes to the inferior planets-A new dawn for NEO and IEO detection technology demonstration from heliocentric orbits interior to the earth's?
}

\author{
Jan Thimo Grundmann ${ }^{\mathrm{a}, *}$, Stefano Mottola ${ }^{\mathrm{b}}$, Maximilian Drentschew ${ }^{\mathrm{f}}$, \\ Martin Drobczyk ${ }^{\mathrm{a}}$, Ralph Kahle ${ }^{\mathrm{c}}$, Volker Maiwald ${ }^{\mathrm{d}}$, Dominik Quantius ${ }^{\mathrm{d}}$, \\ Paul Zabel ${ }^{\mathrm{d}}$, Tim van Zoest ${ }^{\mathrm{e}}$ \\ ${ }^{a}$ DLR German Aerospace Center-Institute of Space Systems_Department of Satellite Systems, Robert-Hooke-Straße 7, 28359 Bremen, Germany \\ ${ }^{\mathrm{b}}$ DLR German Aerospace Center-Institute of Planetary Research_Department Asteroids and Comets, Rutherfordstraße 2, 12489 Berlin, Germany \\ ${ }^{\mathrm{c}}$ DLR German Aerospace Center_Space Operations and Astronaut Training_Space Flight Technology Department, 82234 \\ Oberpfaffenhofen-Wesseling, Germany \\ ${ }^{\mathrm{d}}$ DLR German Aerospace Center-Institute of Space Systems_Department System Analysis Space Segment (SARA), Robert-Hooke-Straße \\ 7, 28359 Bremen, Germany \\ ${ }^{\mathrm{e}}$ DLR German Aerospace Center-Institute of Space Systems_-Department of Exploration Systems, Robert-Hooke-Straße 7, 28359 Bremen, Germany \\ ${ }^{\mathrm{f}}$ ZFT Zentrum für Telematik, Allesgrundweg 12, 97218 Gerbrunn, Germany
}

\section{A R T I C L E I N F O}

\section{Article history:}

Received 31 January 2012

Received in revised form

24 August 2012

Accepted 1 October 2012

Available online 13 November 2012

Keywords:

IEO

NEO

VENUS EXPRESS

MESSENGER

Akatsuki

BepiColombo

\begin{abstract}
A B S T R A C T
Recent years have seen a renewed interest in exploration of the interior of the solar system. A number of missions are currently under way, in planning as well as in space, with the primary goal to expand our knowledge on the planets Mercury and Venus. Chemical propulsion missions to Mercury in particular require an extended cruise phase prior to arrival at their destination, usually involving multiple planetary fly-by manoeuvres and many revolutions in heliocentric orbit. The difficulties in discovering and tracking small objects interior to Earth's orbit, mainly due to unfavourable viewing geometry as well as atmospheric interference, have long been noted by the solar system science and planetary defence communities. Space probes in the interior of the solar system are in a position to observe objects near or interior to Earth's orbit in favourable opposition geometry. They are also usually free from planet-related interference, at least while in cruise, and often can be while in planetary eclipse.

Dedicated search and survey missions to look for Near and Inner Earth Objects (NEO, IEO) from the vicinity of Earth or low Earth orbit are being planned. In this article, the ad-hoc available as well as near-term planned in-situ capabilities of the optical instrument payloads of space probes to Venus and Mercury are compiled from publications by the respective instrument teams. The small-object detection capabilities of cameras and spectrographs in opposition geometry are estimated by a common method, using data from comparable instruments to supplement missing information where necessary. The on-board cameras are classified according to their small-object detection potential in a technology demonstration of asteroid detection from a heliocentric orbit substantially interior to Earth's.
\end{abstract}

(c) 2012 IAA. Published by Elsevier Ltd. All rights reserved.

\footnotetext{
* Corresponding author. Tel.: +4942124420 1107; fax: +49421244201120.

E-mail addresses: jan.grundmann@dlr.de (J.T. Grundmann), stefano.mottola@dlr.de (S. Mottola), maximilian.drentschew@telematikzentrum.de (M. Drentschew), martin.drobczyk@dlr.de (M. Drobczyk), ralph.kahle@dlr.de (R. Kahle), volker.maiwald@dlr.de (V. Maiwald), dominik.quantius@dlr.de (D. Quantius), paul.zabel@dlr.de (P. Zabel), tim.zoest@dlr.de (T. van Zoest).
} 


\section{Introduction}

With the launch of MESSENGER [1] and Venus EXPRESS [2], a new wave of exploration of the inner solar system has begun. Noting the growing number of probes to the inner solar system, it is proposed to connect the expertise of the respective spacecraft teams and the NEO and IEO survey community to best utilise the extended cruise phases and to provide additional data return in support of pure science as well as planetary defence.

Several missions to Venus and Mercury are planned to follow in this decade. Increased interest in the inferior planets is accompanied by several missions designed to study the Sun and the interplanetary medium (IPM) from a position near or in Earth orbit, such as the STEREO probes [3] and SDO [4]. These augment established solar observation capabilities at the Sun-Earth L1 Lagrangian point such as the SOHO $[5,6]$ spacecraft. Thus, three distinct classes of spacecraft operate or observe interior to Earth's orbit. All these spacecrafts carry powerful multispectral cameras optimised for their respective primary targets.

MESSENGER has meanwhile ended its 61/2-year interplanetary cruise on March 18th, 2011, to enter Mercury orbit, but a similarly extended cruise with several gravityassists awaits the European Mercury mission BepICoLombo [7]. Unfortunately, the automatic abort of the orbit insertion manoeuvre in December 2010 has also left AKATSUKI (a.k.a. Venus Climate Orbiter (VCO), Planet-C) [8-10] stranded in heliocentric orbit. After an unintended flyby, the probe has in November 2011 been manoeuvred to catch up with Venus in 2015 or 2016. Meanwhile, it stays mostly interior to Venus in a planet-leading orbit. [11]

In addition to the study of comets and their interaction with the IPM, observations of small bodies akin to those carried out by outer solar system probes are occasionally attempted with the equipment available. The study of structures in the interplanetary dust (IPD) cloud has been a science objective during the cruise phase of the Japanese Venus probe АкатsUкI from Earth to Venus. IPD observations in the astronomical H-band $(1.65 \mu \mathrm{m})$ are supported by its IR2 camera down to $1.5 \mu \mathrm{W} / \mathrm{m}^{2} \mathrm{sr}$ in single $2 \mathrm{~min}$ exposures. In the same setting, point sources of 13 mag can be detected. Obviously, a number of large asteroids exceed this threshold.

The EARTHGUARD-I study [12-14], completed in 2003 by Kayser-Threde and the DLR Institute of Planetary Research under ESA contract, proposed a dedicated steerable telescope in the size range $20-35 \mathrm{~cm}$ and CCD camera payload on a probe to the inner solar system, to detect Near-Earth and Inner-Earth Objects (NEOs, IEOs) in favourable opposition geometry. A ride-share on a Mercury orbiter and a dedicated low-thrust propulsion spacecraft to a heliocentric 0.5 AU orbit were studied. A similarsized telescope was under development for the AsTEROIDFINDER [15], a low Earth orbit small satellite project of DLR. The Canadian small satellite NEOSSAT [16] carries a smaller telescope for a shared space situational awareness and IEO search mission; it is currently awaiting launch as a secondary payload. Four large spacecraft concepts, using visible light or mid-infrared telescopes in low-elongation or opposition mode, have recently been studied, also with the goal to broaden the target base for a manned NEO mission [17]; the NEOCAM [18] proposal has been selected for a technology development study phase in 2011. A NEO survey using the DEEP IMPACT fly-by bus is currently being proposed. [19] After its primary mission of guiding an impactor vehicle to comet 9P/Tempel [20], this spacecraft now has already been re-purposed once for the EPOXI mission including such diverse topics as a close fly-by of 103P/Hartley, long-distance observations of 2009 P1 Garradd over $1.4 \mathrm{AU}$, and the observation to characterise known exoplanets lightyears away. [21] A NEO survey would constitute a second re-use of flying hardware [22], an approach that may seem obvious given the effort required to develop any mission proposal into a flying spacecraft but nevertheless is uncommon even for Earthorbiting spacecraft. [23]

In the following, the technical feasibility of a number of asteroid observation scenarios involving spacecraft and targets interior to Earth's orbit is assessed based on the latest available spacecraft information and asteroid population models. A rough estimate of the required effort in terms of ground-based spacecraft operations and onboard resources is given for selected representative scenarios. Given the number of dedicated IEO survey missions currently under study or in development, the focus is on near-term pathfinder scenarios using spacecraft which are already in space or expected to be launched soon.

\subsection{Relevant small solar system bodies}

Small solar system bodies (SSSB) which are classified as Near-Earth Objects (NEO) approach the Sun to $\mathrm{q}_{\mathrm{NEA}}=1.3$ Astronomical Units (AU) or less. The Near Earth Asteroids (NEA) among them are divided into four classes according to the relationship of their orbital parameters perihelion $q_{\mathrm{A}}$, semi-major axis $a_{\mathrm{A}}$, and aphelion $Q_{\mathrm{A}}$ with the corresponding parameters of Earth's orbit, $q_{\oplus}=0.983 \mathrm{AU}, a_{\oplus}=1 \mathrm{AU}, Q_{\oplus}=1.017 \mathrm{AU}$.

- Amor class: $a_{\oplus}<a_{\mathrm{A}} \quad$;

- Apollo class: $a_{\oplus}<a_{\mathrm{A}}$

- Aten class: $a_{\mathrm{A}}<a_{\oplus}$

- Atira class: $a_{\mathrm{A}}<a_{\oplus}$

$$
\begin{array}{cr}
; & Q_{\oplus}<q_{\mathrm{A}}<q_{\mathrm{NEA}} \\
; & q_{\mathrm{A}}<Q_{\oplus} \\
; & Q_{\oplus}<Q_{\mathrm{A}}<q_{\oplus}
\end{array}
$$

A separate class definition exists for Potentially Hazardous Objects (PHO) which have an absolute magnitude $H \leq 22.0$, therefore inferred to be larger than $140 \mathrm{~m}$ estimated diameter $(\varnothing)$, and are on an orbit which approaches the Earth's to within 0.05 AU or less. [38] Note that the thresholds for perihelia and aphelia of the four 'A' NEA classes do not imply automatically that a close approach geometry exists. Also, the fraction of comets (NEC, PHC) is very small in NEOs [24].

Due to the location of the Earth within the cloud of NEOs, geometrical observation conditions are rarely favourable, especially for objects that cross the Earth's orbit. Of these Earth-Crossing Asteroids (ECA), Apollo class objects have orbits mostly outside the Earth's orbit, 
and Aten class objects mostly within. Favourable observation conditions only exist while they are near aphelion, outside the Earth's orbit and while the Earth passes on the inside, closer to the Sun. For ground-based observations, most of the celestial sphere is inaccessible due to the additional interference of the Sun and Earth's atmosphere, in the form of the bright day-time sky, and extinction close to the horizon. Only during brief periods of opposition, for which aphelion of the object of interest and conjunction with the Earth have to coincide, detection probability within the capabilities of given equipment is high. Atira class NEAs, also known as Inner-Earth Objects (IEO) orbiting the Sun entirely Interior to Earth's Orbit are still harder to detect than Aten class objects. Only 11 of an estimated > 1000 IEOs larger than $\varnothing 100 \mathrm{~m}$ are presently known, all but one of them in borderline Aten-like orbits.

\subsection{In the beginning-the EARTHGUARD I study}

EARTHGUARD I was a mission proposal studied under an ESA contract by Kayser-Threde with the DLR Institute of Planetary Research in July 2002 to January 2003. The mission concept revolved around the idea of sending a NEO detection telescope to an inner solar system orbit to observe NEOs, ECAs, and IEOs in opposition where they are easiest to detect by observational geometry, and without additional interference due to Earth's atmosphere.

The space segment studied included two design options, a separate spacecraft or an instrument added to another space probe, depending on available launches and at the time planned missions:

- instrument-only option:

- rideshare of the telescope mounted on an independent pointing platform on a space probe

- flight to Mercury studied, based on BePI-Colombo as then envisaged

- independent spacecraft option:

○ dedicated launch to $\sim 0.5 \mathrm{AU}$ heliocentric orbit

study focus on the use of advanced low-thrust propulsion in interplanetary space

- e.g. solar sail from GTO rideshare with own kick stage.

The EARTHGUARD I mission was to be equipped with a $\varnothing$ $20 \ldots 35 \mathrm{~cm}$ reflector telescope using a $2048^{2}$ pixel resolution CCD camera augmented by 3 in-field star tracking sensors. A mission duration of 400 days was envisaged, and the detection of approximately $80 \%$ of all NEAs $>\varnothing$ $1 \mathrm{~km}$ expected in this time. [12-14].

The IEO search component of EARTHGUARD I evolved into the AsTEROIDFINDER project in the German national 'Kompaktsatellit' programme of the DLR Research and Development programmatic branch. [15] The EARTHGUARD I telescope design study baseline was, for some time and with extensive modifications, held as a fall-back option for the more advanced telescope and sensor concept that were to be used in AsTEROIDFINDER.

\section{Deep space probes and cameras in the region of the inferior planets}

\subsection{Missions between earth and sun}

The past decade has seen a renaissance of exploration of the inferior planets, Mercury and Venus. Currently, three missions equipped with various cameras and spectrometers operate in the interior solar system; Venus EXPRESS, MESSENGER, and AKATSUKI. More planetary research missions are planned for this decade, including the ESA mission BepIColombo. Also, solar research missions equipped with cameras now venture into interplanetary space, for example the pair of STEREO spacecraft orbiting the Sun ahead and behind the Earth in very similar orbits to it, to provide a complete coverage of the Sun in cooperation with Earth-based observatories, Earth-orbiting satellites such as SDO, and solar probes stationed at the Sun-Earth Langrange point L1 such as SOHO.

A notable addition to this array is the Deep Impact fly-by bus which carries a suite of two very powerful cameras and a near-to-mid-IR spectrometer. Unlike the cameras of these other missions, its remote sensing suite was optimised for small-object observations in a close and fast fly-by for science objectives as well as from a long distance for the purpose of optical navigation. [20] Although it is on an Earth-like, Earth-crossing orbit extending only slightly into the interior solar system, its cameras offer a useful reference point in optical design and performance when compared to those of the planetary probes designed to observe very bright and extended objects.

\subsection{Planetary spacecraft and cameras}

The following planetary research space probes equipped with cameras and sensitive spectrometers are currently active on orbits significantly interior to the Earth's or in the advanced stages of planning:

- Venus Express [25] - Venus Monitoring Camera (VMC) [26]

- $17.5^{\circ}$ Field of View (FoV), $13 \mathrm{~mm}$ focal length (f.l.), $f / 5$ wide-angle camera

- Visible and Infrared Thermal Imaging Spectrometer (VIRTIS) [27]

- 64 mrad FoV, $f / 5.6$ UV/VIS channel, 2 nm resolution

- 64 mrad FoV, $f / 3.2$ near to mid IR channel, $10 \mathrm{~nm}$ resolution

- 0.45 - 2.25 mrad FoV, f/2 mid IR channel, $3 \mathrm{~nm}$ resolution

- Spectroscopy for Investigation of Characteristics of the Atmosphere of Venus (SPICAV) [28]

- $1 \cdot 3.16^{\circ}$ FoV, $118 \mathrm{~mm}$ f.l. UV channel, $0.55 \mathrm{~nm}$ resolution

- $2^{\circ}$ FoV, $40 \mathrm{~mm}$ f.l. VIS to near IR channel, $0.55 \mathrm{~nm}$ resolution

- 180 mm f.l., f/4 mid IR channel 
- MESSENGER

- Mercury Dual Imaging System (MDIS) [29]

- $10.5^{\circ} \mathrm{FoV}, 78 \mathrm{~mm}$ f.l., multispectral Wide Angle Camera (WAC)

- $1.5^{\circ}$ FoV, $550 \mathrm{~mm}$ f.l., $f / 22$ monochromatic Narrow Angle Camera (NAC)

- Mercury Atmospheric and Surface Composition Spectrometer (MASCS) [31]

- $0.04^{\circ} \cdot 1^{\circ} \mathrm{FoV}, 258 \mathrm{~mm}$ f.l., $f / 5$ front-end telescope

- resolution $0.6 \mathrm{~nm} \mathrm{UV} / \mathrm{VIS}, 4.7 \mathrm{~nm}$ resolution IR

- АкатsUкі $[8,9,32]$

- common FoV $12^{\circ}$

Ultraviolet Imager (UVI)

Lightning and Airglow Camera (LAC, FoV 16 ${ }^{\circ}$ )

- 8.8 photodiode array, filters for airglow and lightning emission lines, split into

- one 4.8 lightning detector ( $50 \mathrm{kHz}$, by pretrigger) and

- four 1 . 8 spectral and background fields ( $0.05 \mathrm{~Hz}$, nominal read-out cycle)

- $1 \mu \mathrm{m}$ Infrared Camera (IR1) [33]

- $84 \mathrm{~mm}$ f.l., $f / 4$, multispectral near IR camera

- $2 \mu \mathrm{m}$ Infrared Camera (IR2)

- $84 \mathrm{~mm}$ f.l., $f / 4$, multispectral mid-IR camera, including IPD filter channel (cf.[34])

- Longwave Infrared Camera (LIR, FoV $16.4^{\circ} \cdot 12.4^{\circ}$ )

- $f / 1.4$, thermal IR camera, $328 \cdot 248$ bolometer array

- BeprColombo

- Spectrometers and Imagers for MPO BepiColombo Integrated Observatory System (SIMBIO-SYS) [35]

- $1.47^{\circ} \mathrm{FoV}, 800 \mathrm{~mm}$ f.l., $f / 8$ High Resolution Imaging Channel (HRIC)

- $5.3^{\circ} \mathrm{FoV}, 90 \mathrm{~mm}$ f.l., $f / 6$ multispectral Stereo Channel (STC)

- 0.25 . $64 \mathrm{mrad}$ FoV, $160 \mathrm{~mm}$ f.l., $f / 6.4$ Visible and Infrared Hyperspectral Imager (VIHI), $6.25 \mathrm{~nm}$ resolution

Mercury Radiometer and Thermal Infrared Spectrometer (MERTIS) [37]

- $4^{\circ} \mathrm{FoV}, 50 \mathrm{~mm}$ f.l., $f / 2$ mid to thermal IR spectrometer, $90 \mathrm{~nm}$ resolution

Note that there is a general similarity with respect to the FoV classes of the cameras used in many planetary spacecraft which suggests that Narrow-, Medium-, and Wide-Angle Cameras of approximately $1.5^{\circ}, 5^{\circ}$, and $15^{\circ}$ FoV are favoured, with 2 out of 3 usually present. Table 1 gives an overview of key camera parameters, also including the camera systems of the DeEp Impact mission for comparison:

\section{NEO opposition detection capability of existing and near-term space assets}

\subsection{Methods}

To evaluate the general feasibility of the detection of NEOs from probes in the region of the inferior planets, the performance of the camera systems was studied based on published camera system parameters. Wherever data was lacking, information from comparable systems was substituted. The NEO target was assumed as a point source with the spectrum of a G0 star, seen from the orbit of Venus at $0.7 \mathrm{AU}$ in opposition. A Signal-to-Noise (SNR) of 5 was assumed for object detection.

\subsection{Results for selected spacecraft cameras}

The following list gives a brief overview of the capabilities and possible NEO detection feasibility demonstration scenarios for the respective spacecraft and cameras which are marked by light green lines in Table 1 :

- Venus Express

- Venus Monitoring Camera (VMC) - Fig. 1

- filter: F4 (VIS) - $513 \mathrm{~nm}, 50 \mathrm{~nm}$ bandwidth

- can not detect the brightest main belt asteroid, (4) Vesta, even under the most favourable conditions

- even large NEOs would need to come exceptionally close to become detectable

- sensor large, but shared between 4 instrument channels

- no useful potential for EARThGUARD I-like technology demonstrations

- observation of very bright comets may occasionally become possible

- MESSENGER

- Mercury Dual Imaging System Narrow Angle Camera (MDIS NAC)—Fig. 2

- filter fixed monochromatic-750 nm, $100 \mathrm{~nm}$ bandwidth

- stated point source sensitivity: optical navigation detection limit $M_{\mathrm{v}}=8.3 \mathrm{mag}$ or $14 \mathrm{DN}$ at signal/read noise ratio $=7$, in $10 \mathrm{~s}$ exposure, $2^{2}$ pixel sum, at 70\% ensquared energy [29]

- achieved point source limiting magnitude in cruise: $M_{\mathrm{v}}=8.3 \mathrm{mag}$ without any specialprocessing, $M_{\mathrm{v}}=9.5 \ldots 10 \mathrm{mag}$ with multiple exposures and post-processing [30]

o given the limiting magnitude of the system, even large NEOs would need to come very close to become detectable

- large, $>100 \mathrm{~km}$ mainbelt asteroids can be detected

o a limited Earthguard I-like technology demonstration is feasible

- Акатsuki

- $1 \mu \mathrm{m}$ Infrared Camera (IR1)-Fig. 3

- filter: night-side- $900 \mathrm{~nm}, 30 \mathrm{~nm}$ bandwidth

$2 \mu \mathrm{m}$ Infrared Camera (IR2)

- filter: Zodiacal IPD-1650 nm, $30 \mathrm{~nm}$ bandwidth

- stated point source sensitivity: 13 mag H-band in single exposure, 2 min integration

- long integration, wide FOV and many similarbandwidth filters are available

- large NEOs would need to come exceptionally close to become detectable

- the largest, $>200 \mathrm{~km}$ mainbelt asteroids can be detected using the IR1 camera

- the spacecraft is in a long cruise period to a reencounter with Venus at $\sim 0.7 \mathrm{AU}$ 
Table 1

Camera parameters of spacecraft in the interior solar system.

\begin{tabular}{|c|c|c|c|c|c|c|c|c|c|c|c|}
\hline Spacecraft & Instrument & $\begin{array}{l}\text { FOV cross-track } \\
\text { (deg.) }\end{array}$ & $\begin{array}{l}\text { FOV along-track } \\
\text { (deg.) }\end{array}$ & $\begin{array}{l}\text { FOV cross-track } \\
\text { (mrad) }\end{array}$ & $\begin{array}{l}\text { FOV along-track } \\
\text { (mrad) }\end{array}$ & $\begin{array}{l}\text { Focal length } \\
(\mathrm{mm})\end{array}$ & $\begin{array}{l}f \text { no. } \\
(f / \mathrm{n})\end{array}$ & $\begin{array}{l}\text { Sensor pixel } \\
\text { (column) }\end{array}$ & $\begin{array}{l}\text { Sensor pixel } \\
\text { (line) }\end{array}$ & $\begin{array}{l}\text { Pixel pitch } \\
(\mu \mathrm{m})\end{array}$ & $\begin{array}{l}\text { A/D depth } \\
\text { (bits) }\end{array}$ \\
\hline Akatsuki & Ultraviolet Imager (UVI) & 12 & & & & & & 1024 & 1024 & 13 & 12 \\
\hline Akatsuki & Ultraviolet Imager (UVI) & 12 & & & & & & 1024 & 1024 & 13 & 12 \\
\hline Akatsuki & $\begin{array}{l}\text { Lightning and Airglow } \\
\text { Camera (LAC) }\end{array}$ & 16 & & & & & & 8 & 2 & 2000 & \\
\hline Akatsuki & LAC & 16 & & & & & & 8 & 1 & 2000 & \\
\hline Akatsuki & LAC & 16 & & & & & & 8 & 1 & 2000 & \\
\hline Akatsuki & LAC & 16 & & & & & & 8 & 4 & 2000 & \\
\hline Akatsuki & $1 \mu \mathrm{m}$ Camera (IR1) & 12 & & & & 84.2 & 8 & 1024 & 1024 & 17 & 16 \\
\hline Akatsuki & $1 \mu \mathrm{m}$ Camera (IR1) & 12 & & & & 84.2 & 8 & 1024 & 1024 & 17 & 16 \\
\hline Akatsuki & $1 \mu \mathrm{m}$ Camera (IR1) & 12 & & & & 84.2 & 8 & 1024 & 1024 & 17 & 16 \\
\hline Akatsuki & $1 \mu \mathrm{m}$ Camera (IR1) & 12 & & & & 84.2 & 8 & 1024 & 1024 & 17 & 16 \\
\hline Akatsuki & $2 \mu \mathrm{m}$ Camera (IR2) & 12 & & & & 84.2 & 4 & 1024 & 1024 & 17 & 16 \\
\hline Akatsuki & $2 \mu \mathrm{m}$ Camera (IR2) & 12 & & & & 84.2 & 4 & 1024 & 1024 & 17 & 16 \\
\hline Akatsuki & $2 \mu \mathrm{m}$ Camera (IR2) & 12 & & & & 84.2 & 4 & 1024 & 1024 & 17 & 16 \\
\hline Akatsuki & $2 \mu \mathrm{m}$ Camera (IR2) & 12 & & & & 84.2 & 4 & 1024 & 1024 & 17 & 16 \\
\hline Akatsuki & $2 \mu \mathrm{m}$ Camera (IR2) & 12 & & & & 84.2 & 4 & 1024 & 1024 & 17 & 16 \\
\hline Akatsuki & $\begin{array}{l}\text { Longwave infrared Camera } \\
\text { (LIR) }\end{array}$ & 16.4 & 12.4 & & & & 1.4 & 328 & 248 & 37 & \\
\hline BepiColombo & SIMBIO-SYS HRIC & 1.47 & & & & 800 & 8 & 2048 & 2048 & 10 & \\
\hline BepiColombo & SIMBIO-SYS HRIC & 1.47 & & & & 800 & 8 & 2048 & 2048 & 10 & \\
\hline BepiColombo & SIMBIO-SYS HRIC & 1.47 & & & & 800 & 8 & 2048 & 2048 & 10 & \\
\hline BepiColombo & SIMBIO-SYS HRIC & 1.47 & & & & 800 & 8 & 2048 & 2048 & 10 & \\
\hline BepiColombo & SIMBIO-SYS STC & 5.3 & 4.6 & & & 90 & 6 & 2048 & 2048 & 10 & \\
\hline BepiColombo & SIMBIO-SYS STC & 5.3 & 4.6 & & & 90 & 6 & 2048 & 2048 & 10 & \\
\hline BepiColombo & SIMBIO-SYS STC & 5.3 & 4.6 & & & 90 & 6 & 2048 & 2048 & 10 & \\
\hline BepiColombo & SIMBIO-SYS STC & 5.3 & 4.6 & & & 90 & 6 & 2048 & 2048 & 10 & \\
\hline BepiColombo & SIMBIO-SYS STC & 5.3 & 4.6 & & & 90 & 6 & 2048 & 2048 & 10 & \\
\hline BepiColombo & SIMBIO-SYS VIHI & & & 64 & 0.25 & 160 & 6.4 & 256 & 256 & 40 & 14 \\
\hline BepiColombo & MERTIS & 4 & & & & 50 & 2 & 160 & 120 & 35 & \\
\hline BepiColombo & MERTIS & 4 & & & & 50 & 2 & 15 & 2 & 200 & \\
\hline MESSENGER & MDIS NAC & 1.493 & & & & 550.3 & 22 & 1024 & 1024 & 14 & 12 \\
\hline MESSENGER & MDIS WAC & 10.54 & & & & 77.96 & & 1024 & 1024 & 14 & 12 \\
\hline MESSENGER & MDIS WAC & 10.54 & & & & 77.96 & & 1024 & 1024 & 14 & 12 \\
\hline MESSENGER & MDIS WAC & 10.54 & & & & 77.96 & & 1024 & 1024 & 14 & 12 \\
\hline MESSENGER & MDIS WAC & 10.54 & & & & 77.96 & & 1024 & 1024 & 14 & 12 \\
\hline MESSENGER & MDIS WAC & 10.54 & & & & 77.96 & & 1024 & 1024 & 14 & 12 \\
\hline MESSENGER & MDIS WAC & 10.54 & & & & 77.96 & & 1024 & 1024 & 14 & 12 \\
\hline MESSENGER & MDIS WAC & 10.54 & & & & 77.96 & & 1024 & 1024 & 14 & 12 \\
\hline MESSENGER & MDIS WAC & 10.54 & & & & 77.96 & & 1024 & 1024 & 14 & 12 \\
\hline MESSENGER & MDIS WAC & 10.54 & & & & 77.96 & & 1024 & 1024 & 14 & 12 \\
\hline MESSENGER & MDIS WAC & 10.54 & & & & 77.96 & & 1024 & 1024 & 14 & 12 \\
\hline MESSENGER & MDIS WAC & 10.54 & & & & 77.96 & & 1024 & 1024 & 14 & 12 \\
\hline MESSENGER & MDIS WAC & 10.54 & & & & 77.96 & & 1024 & 1024 & 14 & 12 \\
\hline MESSENGER & MASCS & & & & & 257.6 & 5 & & & & \\
\hline MESSENGER & MASCS UVVS & 1 & 0.04 & & & 125 & & 1 & 1 & 6000 & \\
\hline MESSENGER & MASCS UVVS & 1 & 0.04 & & & 125 & & 1 & 1 & 10000 & \\
\hline MESSENGER & MASCS UVVS & 1 & 0.04 & & & 125 & & 1 & 1 & 10000 & \\
\hline MESSENGER & MASCS VIRS & 0.023 & & & & 210 & & 256 & 1 & 50 & 16 \\
\hline
\end{tabular}


Table 1 (continued)

\begin{tabular}{|c|c|c|c|c|c|c|c|c|c|c|c|}
\hline Spacecraft & Instrument & $\begin{array}{l}\text { FOV cross-track } \\
\text { (deg.) }\end{array}$ & $\begin{array}{l}\text { FOV along-track } \\
\text { (deg.) }\end{array}$ & $\begin{array}{l}\text { FOV cross-track } \\
\text { (mrad) }\end{array}$ & $\begin{array}{l}\text { FOV along-track } \\
\text { (mrad) }\end{array}$ & $\begin{array}{l}\text { Focal length } \\
(\mathbf{m m})\end{array}$ & $\begin{array}{r}f \text { no. } \\
(f / \mathrm{n})\end{array}$ & $\begin{array}{l}\text { Sensor pixel } \\
\text { (column) }\end{array}$ & $\begin{array}{l}\text { Sensor pixel } \\
\text { (line) }\end{array}$ & $\begin{array}{l}\text { Pixel pitch } \\
(\mu \mathrm{m})\end{array}$ & $\begin{array}{l}\begin{array}{l}\text { A/D depth } \\
\text { (bits) }\end{array}\end{array}$ \\
\hline $\begin{array}{l}\text { MESSENGER } \\
\text { MESSENGER } \\
\text { MESSENGER }\end{array}$ & $\begin{array}{l}\text { MASCS VIRS } \\
\text { visible-infrared } \\
\text { spectrometer } \\
\text { Visible-infrared } \\
\text { spectrometer }\end{array}$ & 0.023 & & & & 210 & & 512 & 1 & 50 & \\
\hline $\begin{array}{l}\text { Venus Express } \\
\text { (VEx) }\end{array}$ & SPICAV SUV & 1 & 3.16 & & & 118.13 & & 384 & 288 & 23 & \\
\hline $\begin{array}{l}\text { Venus Express } \\
\text { (VEx) }\end{array}$ & SPICAV SIR & 2 & & & & & & 2 & 1 & 2400 & \\
\hline $\begin{array}{l}\text { Venus Express } \\
\text { (VEx) }\end{array}$ & SPICAV SIR & 2 & & & & 40 & & 2 & 1 & 2000 & 12 \\
\hline $\begin{array}{l}\text { Venus Express } \\
\text { (VEx) }\end{array}$ & SPICAV SIR & 2 & & & & 40 & & 2 & 1 & 500 & 12 \\
\hline $\begin{array}{l}\text { Venus Express } \\
\text { (VEx) }\end{array}$ & SPICAV SOIR & & & & & 180 & 4 & 320 & 256 & 30 & \\
\hline $\begin{array}{l}\text { Venus Express } \\
\text { (VEx) }\end{array}$ & SPICAV SOIR & & & & & 180 & 4 & 320 & 256 & 30 & \\
\hline $\begin{array}{l}\text { Venus Express } \\
\text { (VEx) }\end{array}$ & SPICAV SOIR & & & & & 180 & 4 & 320 & 256 & 30 & \\
\hline $\begin{array}{l}\text { Venus Express } \\
\text { (VEx) }\end{array}$ & VIRTIS-M VIS & & & 64 & 64 & & 5.6 & 508 & 1024 & 19 & 16 \\
\hline $\begin{array}{l}\text { Venus Express } \\
\text { (VEx) }\end{array}$ & VIRTIS-M IR & & & 64 & 64 & & 3.2 & 270 & 438 & 38 & \\
\hline $\begin{array}{l}\text { Venus Express } \\
\text { (VEx) }\end{array}$ & VIRTIS-M IR & & & 64 & 64 & & 3.2 & 270 & 438 & 38 & \\
\hline $\begin{array}{l}\text { Venus Express } \\
\text { (VEx) }\end{array}$ & VIRTIS-M IR & & & 64 & 64 & & 3.2 & 270 & 438 & 38 & \\
\hline $\begin{array}{l}\text { Venus Express } \\
\text { (VEx) }\end{array}$ & VIRTIS-M IR & & & 64 & 64 & & 3.2 & 270 & 438 & 38 & \\
\hline $\begin{array}{l}\text { Venus Express } \\
\text { (VEx) }\end{array}$ & VIRTIS-M IR & & & 64 & 64 & & 3.2 & 270 & 438 & 38 & \\
\hline $\begin{array}{l}\text { Venus Express } \\
\text { (VEx) }\end{array}$ & VIRTIS-M IR & & & 64 & 64 & & 3.2 & 270 & 438 & 38 & \\
\hline $\begin{array}{l}\text { Venus Express } \\
\text { (VEx) }\end{array}$ & VIRTIS-H (IR) & & & 0.45 & 2.25 & & 2.04 & 270 & 438 & 38 & \\
\hline $\begin{array}{l}\text { Venus Express } \\
\text { (VEx) }\end{array}$ & VIRTIS-H (IR) & & & 0.45 & 2.25 & & 2.04 & 270 & 438 & 38 & \\
\hline $\begin{array}{l}\text { Venus Express } \\
\text { (VEx) }\end{array}$ & VIRTIS-H (IR) & & & 0.45 & 2.25 & & 2.04 & 270 & 438 & 38 & \\
\hline $\begin{array}{l}\text { Venus Express } \\
\text { (VEx) }\end{array}$ & vMC & 17.5 & & & & 13 & 7 & 1032 & 1024 & 9 & \\
\hline $\begin{array}{l}\text { Venus Express } \\
\text { (VEx) }\end{array}$ & VMC & 17.5 & & & & 13 & 5 & 1032 & 1024 & 9 & \\
\hline $\begin{array}{l}\text { Venus Express } \\
\text { (VEx) }\end{array}$ & VMC & 17.5 & & & & 13 & 5 & 1032 & 1024 & 9 & \\
\hline $\begin{array}{l}\text { Venus Express } \\
\text { (VEx) }\end{array}$ & vMC & 17.5 & & & & 13 & 5 & 1032 & 1024 & 9 & \\
\hline $\begin{array}{l}\text { Venus Express } \\
\text { (VEx) }\end{array}$ & STR (Star Tracker) & 16.47 & & & & 46 & & 1024 & 1024 & & \\
\hline
\end{tabular}




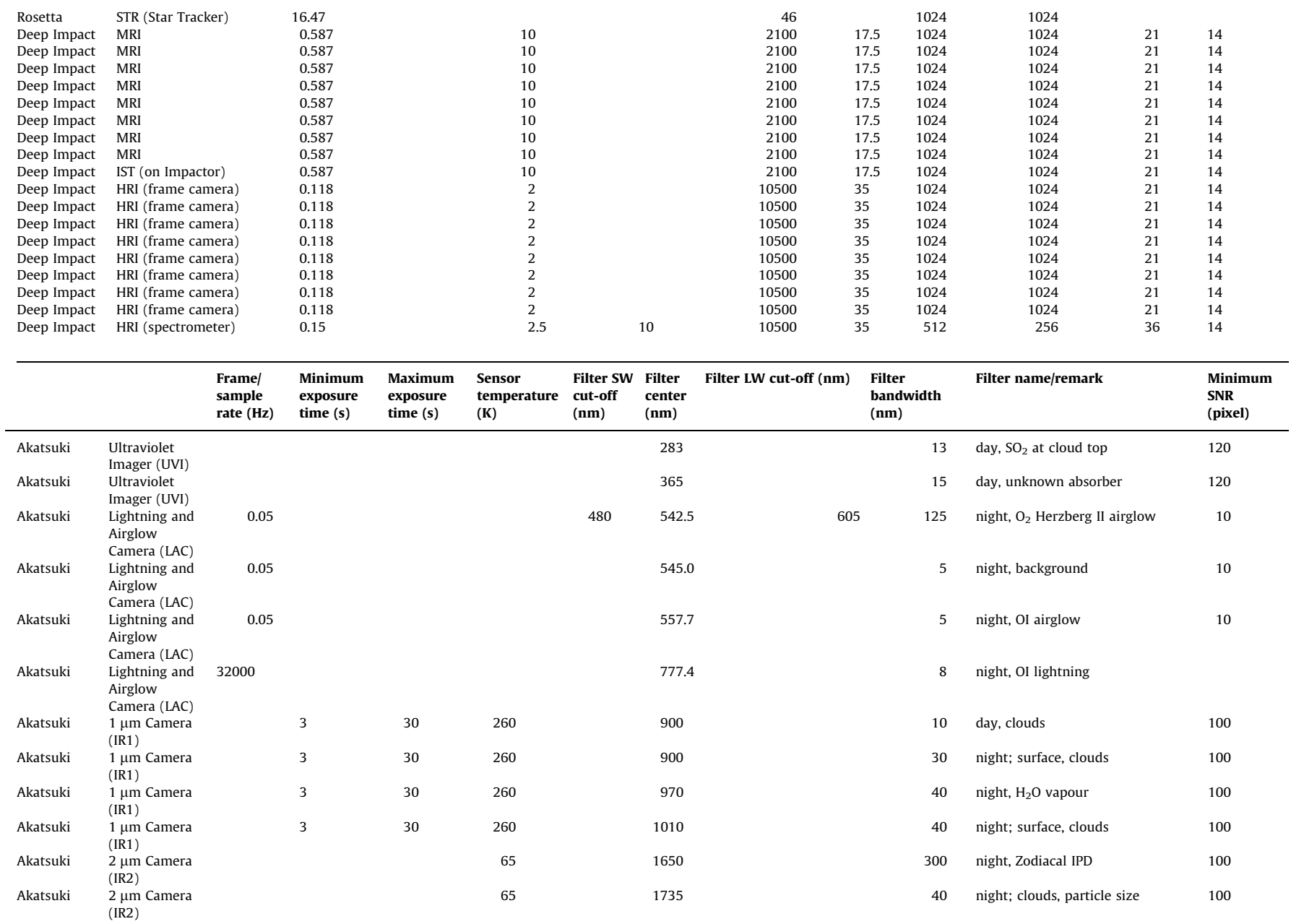


Table 1 (continued)

\begin{tabular}{|c|c|c|c|c|c|c|c|c|c|c|c|}
\hline & & $\begin{array}{l}\text { Frame/ } \\
\text { sample } \\
\text { rate }(\mathrm{Hz})\end{array}$ & $\begin{array}{l}\text { Minimum } \\
\text { exposure } \\
\text { time }(s)\end{array}$ & $\begin{array}{l}\text { Maximum } \\
\text { exposure } \\
\text { time }(s)\end{array}$ & $\begin{array}{l}\text { Sensor } \\
\text { temperature } \\
\text { (K) }\end{array}$ & $\begin{array}{l}\text { Filter SW } \\
\text { cut-off } \\
(\mathbf{n m})\end{array}$ & $\begin{array}{l}\text { Filter } \\
\text { center } \\
(\mathbf{n m})\end{array}$ & Filter LW cut-off (nm) & $\begin{array}{l}\text { Filter } \\
\text { bandwidth } \\
(\mathrm{nm})\end{array}$ & Filter name/remark & $\begin{array}{l}\text { Minimum } \\
\text { SNR } \\
\text { (pixel) }\end{array}$ \\
\hline Akatsuki & $\begin{array}{l}2 \mu \mathrm{m} \text { Camera } \\
\text { (IR2) }\end{array}$ & & & & 65 & & 2020 & & 40 & night, cloud-top height & 100 \\
\hline Akatsuki & $\begin{array}{l}2 \mu \mathrm{m} \text { Camera } \\
\text { (IR2) }\end{array}$ & & & & 65 & & 2260 & & 60 & night; clouds, particle size & 100 \\
\hline Akatsuki & $\begin{array}{l}2 \mu \mathrm{m} \text { Camera } \\
\text { (IR2) }\end{array}$ & & & & 65 & & 2320 & & 40 & night, CO below clouds & 100 \\
\hline Akatsuki & $\begin{array}{l}\text { Longwave } \\
\text { infrared } \\
\text { Camera (LIR) }\end{array}$ & 60 & & & 313 & 8000 & 10000 & 12000 & 4000 & cloud top temperature & \\
\hline BepiColombo & $\begin{array}{l}\text { SIMBIO-SYS } \\
\text { HRIC }\end{array}$ & 200 & & 10 & & & 650 & & 500 & FPAN & 200 \\
\hline BepiColombo & $\begin{array}{l}\text { SIMBIO-SYS } \\
\text { HRIC }\end{array}$ & 200 & & 10 & & & 550 & & 40 & F550 & 200 \\
\hline BepiColombo & $\begin{array}{l}\text { SIMBIO-SYS } \\
\text { HRIC }\end{array}$ & 200 & & 10 & & & 750 & & 40 & F700 & 200 \\
\hline BepiColombo & $\begin{array}{l}\text { SIMBIO-SYS } \\
\text { HRIC }\end{array}$ & 200 & & 10 & & & 880 & & 40 & F880 & 200 \\
\hline BepiColombo & $\begin{array}{l}\text { SIMBIO-SYS } \\
\text { STC }\end{array}$ & 200 & 0.001 & & & & 700 & & 100 & PAN & \\
\hline BepiColombo & $\begin{array}{l}\text { SIMBIO-SYS } \\
\text { STC }\end{array}$ & 200 & 0.001 & & & & 420 & & 20 & & \\
\hline BepiColombo & $\begin{array}{l}\text { SIMBIO-SYS } \\
\text { STC }\end{array}$ & 200 & 0.001 & & & & 550 & & 20 & & \\
\hline BepiColombo & $\begin{array}{l}\text { SIMBIO-SYS } \\
\text { STC }\end{array}$ & 200 & 0.001 & & & & 700 & & 20 & & \\
\hline BepiColombo & $\begin{array}{l}\text { SIMBIO-SYS } \\
\text { STC }\end{array}$ & 200 & 0.001 & & & & 920 & & 20 & & \\
\hline BepiColombo & $\begin{array}{l}\text { SIMBIO-SYS } \\
\text { VIHI }\end{array}$ & 25 & 0.01 & & 220 & 400 & & 2000 & 6.25 & /pixel, hyperspectral & 100 \\
\hline BepiColombo & MERTIS & & & & & 7000 & & 14000 & 90 & $\begin{array}{l}\text { /pixel, hyperspectral ( } 80 \text { channels } \\
90 \ldots 200 \mathrm{~nm} \text { - equally over } \\
7 \ldots 14 \mu \mathrm{m}: 87.5 \mathrm{~nm})\end{array}$ & 100 \\
\hline BepiColombo & MERTIS & & & & & 10000 & & 40000 & & & \\
\hline MESSENGER & MDIS NAC & 1 & 0.001 & 10 & 258.2 & 700 & 750 & 800 & 100 & monochromatic & 200 \\
\hline MESSENGER & MDIS WAC & 1 & 0.001 & 10 & 258.2 & 395 & 700 & 1040 & 600 & 2-clear-panchromatic & 200 \\
\hline MESSENGER & MDIS WAC & 1 & 0.001 & 10 & 258.2 & 420 & 430 & 440 & 18 & 6-violet & 200 \\
\hline MESSENGER & MDIS WAC & 1 & 0.001 & 10 & 258.2 & 465 & 480.4 & 485 & 8.9 & 3-blue & 200 \\
\hline MESSENGER & MDIS WAC & 1 & 0.001 & 10 & 258.2 & 555 & 559.2 & 565 & 4.6 & 4 -green & 200 \\
\hline MESSENGER & MDIS WAC & 1 & 0.001 & 10 & 258.2 & 695 & 628.7 & 705 & 4.4 & 5-red & 200 \\
\hline MESSENGER & MDIS WAC & 1 & 0.001 & 10 & 258.2 & & 698.8 & & 4.4 & 1-far red & 200 \\
\hline MESSENGER & MDIS WAC & 1 & 0.001 & 10 & 258.2 & 745 & 749.0 & 755 & 4.5 & 7-NIR & 200 \\
\hline MESSENGER & MDIS WAC & 1 & 0.001 & 10 & 258.2 & 825 & 828.6 & 835 & 4.1 & $12-\mathrm{NIR}$ & 200 \\
\hline MESSENGER & MDIS WAC & 1 & 0.001 & 10 & 258.2 & 895 & 898.1 & 905 & 4.3 & $10-\mathrm{NIR}$ & 200 \\
\hline MESSENGER & MDIS WAC & 1 & 0.001 & 10 & 258.2 & 945 & 948.0 & 950 & 4.9 & 8-NIR & 200 \\
\hline MESSENGER & MDIS WAC & 1 & 0.001 & 10 & 258.2 & 980 & 996.8 & 1010 & 12.0 & 9-NIR & 200 \\
\hline MESSENGER & MDIS WAC & 1 & 0.001 & 10 & 258.2 & 975 & 1010 & 1045 & 20 & 11-NIR & 200 \\
\hline MESSENGER & MASCS & & & & & & & & & & \\
\hline MESSENGER & MASCS UVVS & & & & & 115 & & 180 & 0.6 & FUV PMT & 100 \\
\hline
\end{tabular}


MESSENGER MASCS UVVS MESSENGER MASCS UVVS MESSENGER MASCS VIRS

MESSENGER MASCS VIRS

$\begin{array}{ll}\text { MESSENGER } & \begin{array}{l}\text { visible-infrared } \\ \text { spectrometer }\end{array}\end{array}$

$\begin{array}{ll} & \text { spectrom } \\ \text { MESSENGER } & \text { Visible- }\end{array}$

infrared

$\begin{array}{ll} & \text { spectrometer } \\ \text { Venus } & \text { SPICAV SUV }\end{array}$

Express
Venus

Express

Venus SPICAV SIR

Express

Venus

Express

Express

Express

Venus

Express

Venus

Express

Venus

Express

Venus

Express

Venus

Express

Venus

Express

Venus

Express

Venus

Express

Venus

Express

Venus

Express

Express

Express

Express

Express

Venus

Express
Venus

Venus

Venus

Express

Rosetta

SPICAV SIR

SPICAV SOIR

SPICAV SOIR

SPICAV SOIR

VIRTIS-M VIS

VIRTIS-M IR

VIRTIS-M IR

VIRTIS-M IR

VIRTIS-M IR

VIRTIS-M IR

VIRTIS-M IR

VIRTIS-H (IR)

VIRTIS-H (IR)

VIRTIS-H (IR)

VMC

VMC

VMC

VMC

STR (Star

Tracker)

STR (Star
270

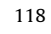

250

650

1050

$10.0 \quad 0.000003$

0.1796

258.2

110

$10.0 \quad 0.000003$

$10.0 \quad 0.000003$

0.02

155

70

70

$1.6203 \quad 0.000504$

32.4823

$1.6203 \quad 0.000504$

32.4823

$1.6203 \quad 0.000504$

32.4823

$1.6203 \quad 0.000504$

32.4823

250

950

900

1200

2400

3600

4300

2000

2000

3900

350

$\begin{array}{rrl}320 & 0.6 & \text { MUV PMT } \\ 600 & 0.6 & \text { VIS PMT } \\ 1050 & 4.7 & \\ 1450 & 4.7 & \\ \text { Grating } 100 \text { grooves/mm } & \text { MESSENGER } & \text { Visible-infrared spectrometer } \\ \text { Grating } 100 \text { grooves/mm } & \text { MESSENGER } & \text { Visible-infrared spectrometer }\end{array}$

2500

3172

4500

365

513

965

1010
320

1700

1050

1700

$0.55 /$ pixel

$0.45 /$ pixel $0.45 \ldots 1.12 \mathrm{~nm} / \mathrm{pixel}$

$0.42 /$ pixel $0.42 \ldots 1.44 \mathrm{~nm} /$ pixel

$0.55 /$ pixel $0.55 \ldots 1.5 \mathrm{~nm} /$ pixel

11.65

18.87

38.18

$1000 \quad 2 \quad /$ pixel

$5000 \quad 10 \quad /$ pixel

$1600 \quad 10 \quad /$ pixel; 1 st region

$2500 \quad 10 \quad /$ pixel; 2nd region

$3750 \quad 10 \quad /$ pixel; 3rd region

$4400 \quad 10 \quad /$ pixel; 4 th region

$5000 \quad 10 \quad /$ pixel; 5 th region

$5000 \quad 3 \quad /$ pixel

44003 /pixel; 1st region

$5000 \quad 3 \quad /$ pixel; 2nd region

$385 \quad 40 \quad$ F3 (UV)

$560 \quad 50 \quad$ F4 (VIS)

$1030 \quad 20 \quad$ F6 (NIR2)
$990 \quad 70 \quad$ F5 (NR1)
100

100
100 
Table 1 (continued)

\begin{tabular}{|c|c|c|c|c|c|c|c|c|c|c|c|}
\hline & & $\begin{array}{l}\text { Frame/ } \\
\text { sample } \\
\text { rate }(\mathrm{Hz})\end{array}$ & $\begin{array}{l}\text { Minimum } \\
\text { exposure } \\
\text { time }(s)\end{array}$ & $\begin{array}{l}\text { Maximum } \\
\text { exposure } \\
\text { time }(s)\end{array}$ & $\begin{array}{l}\text { Sensor } \\
\text { temperature } \\
\text { (K) }\end{array}$ & $\begin{array}{l}\text { Filter SW } \\
\text { cut-off } \\
\text { (nm) }\end{array}$ & $\begin{array}{l}\text { Filter } \\
\text { center } \\
(\mathbf{n m})\end{array}$ & Filter LW cut-off (nm) & $\begin{array}{l}\text { Filter } \\
\text { bandwidth } \\
\text { (nm) }\end{array}$ & Filter name/remark & $\begin{array}{l}\text { Minimum } \\
\text { SNR } \\
\text { (pixel) }\end{array}$ \\
\hline Deep Impact & MRI & 0.612 & 0 & & 188 & & 309 & & 6.2 & $9(\mathrm{OH}$, coma $)$ & \\
\hline Deep Impact & MRI & 0.612 & 0 & 1048.576 & 188 & & 345 & & 6.8 & 8 (dust, coma) & \\
\hline Deep Impact & MRI & 0.612 & 0 & 1048.576 & 188 & & 387 & & 6.2 & $7(\mathrm{CN}, \mathrm{coma})$ & \\
\hline Deep Impact & MRI & 0.612 & 0 & 1048.576 & 188 & & 514 & & 11.8 & $2(\mathrm{C} 2, \mathrm{coma})$ & \\
\hline Deep Impact & MRI & 0.612 & 0 & 1048.576 & 188 & & 526 & & 5.6 & 3 (dust, coma) & \\
\hline Deep Impact & MRI & 0.612 & 0 & 1048.576 & 188 & 320 & 650 & 1050 & $>700$ & 1,6 (uncoated) & \\
\hline Deep Impact & MRI & 0.612 & 0 & 1048.576 & 188 & & 750 & & 100 & 4 (context) & \\
\hline Deep Impact & MRI & 0.612 & 0 & 1048.576 & 188 & & 950 & & 100 & 5 ( , longpass) & \\
\hline Deep Impact & $\begin{array}{l}\text { IST (on } \\
\text { Impactor) }\end{array}$ & 0.612 & 0 & 1048.576 & $<243$ & & & & & unfiltered & \\
\hline Deep Impact & $\begin{array}{l}\text { HRI (frame } \\
\text { camera) }\end{array}$ & 0.612 & 0 & 1048.576 & 160 & & 350 & & 100 & 4 (shortpass) & 70 \\
\hline Deep Impact & $\begin{array}{l}\text { HRI (frame } \\
\text { camera) }\end{array}$ & 0.612 & 0 & 1048.576 & 160 & & 450 & & 100 & 2 & 70 \\
\hline Deep Impact & $\begin{array}{l}\text { HRI (frame } \\
\text { camera) }\end{array}$ & 0.612 & 0 & 1048.576 & 160 & & 550 & & 100 & 3 & 70 \\
\hline Deep Impact & $\begin{array}{l}\text { HRI (frame } \\
\text { camera) }\end{array}$ & 0.612 & 0 & 1048.576 & 160 & 320 & 650 & 1050 & $>700$ & 1,6 (uncoated) & 70 \\
\hline Deep Impact & $\begin{array}{l}\text { HRI (frame } \\
\text { camera) }\end{array}$ & 0.612 & 0 & 1048.576 & 160 & & 650 & & 100 & 9 & 70 \\
\hline Deep Impact & $\begin{array}{l}\text { HRI (frame } \\
\text { camera) }\end{array}$ & 0.612 & 0 & 1048.576 & 160 & & 750 & & 100 & 7 & 70 \\
\hline Deep Impact & $\begin{array}{l}\text { HRI (frame } \\
\text { camera) }\end{array}$ & 0.612 & 0 & 1048.576 & 160 & & 850 & & 100 & 8 & 70 \\
\hline Deep Impact & $\begin{array}{l}\text { HRI (frame } \\
\text { camera) }\end{array}$ & 0.612 & 0 & 1048.576 & 160 & & 950 & & 100 & 5 (longpass) & 70 \\
\hline Deep Impact & $\begin{array}{l}\text { HRI } \\
\text { (spectrometer) }\end{array}$ & 0.3494 & 2.86 & & $<85$ & 1050 & & 4800 & & $\lambda / \mathrm{d} \lambda \geq 196$ & \\
\hline
\end{tabular}




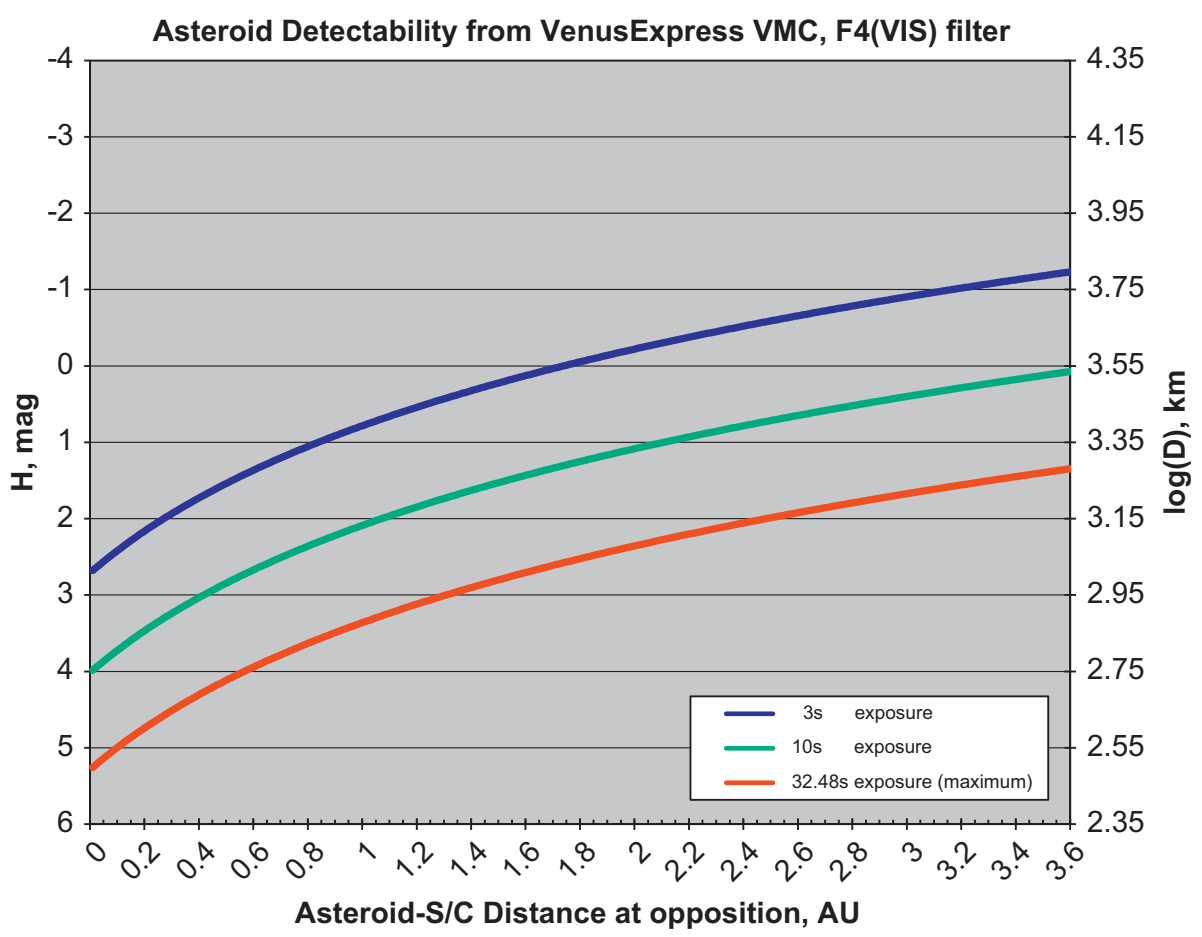

Fig. 1. Detection limit size-distance functions for the Venus ExpREsS VMC channel using the F4(VIS) filter.

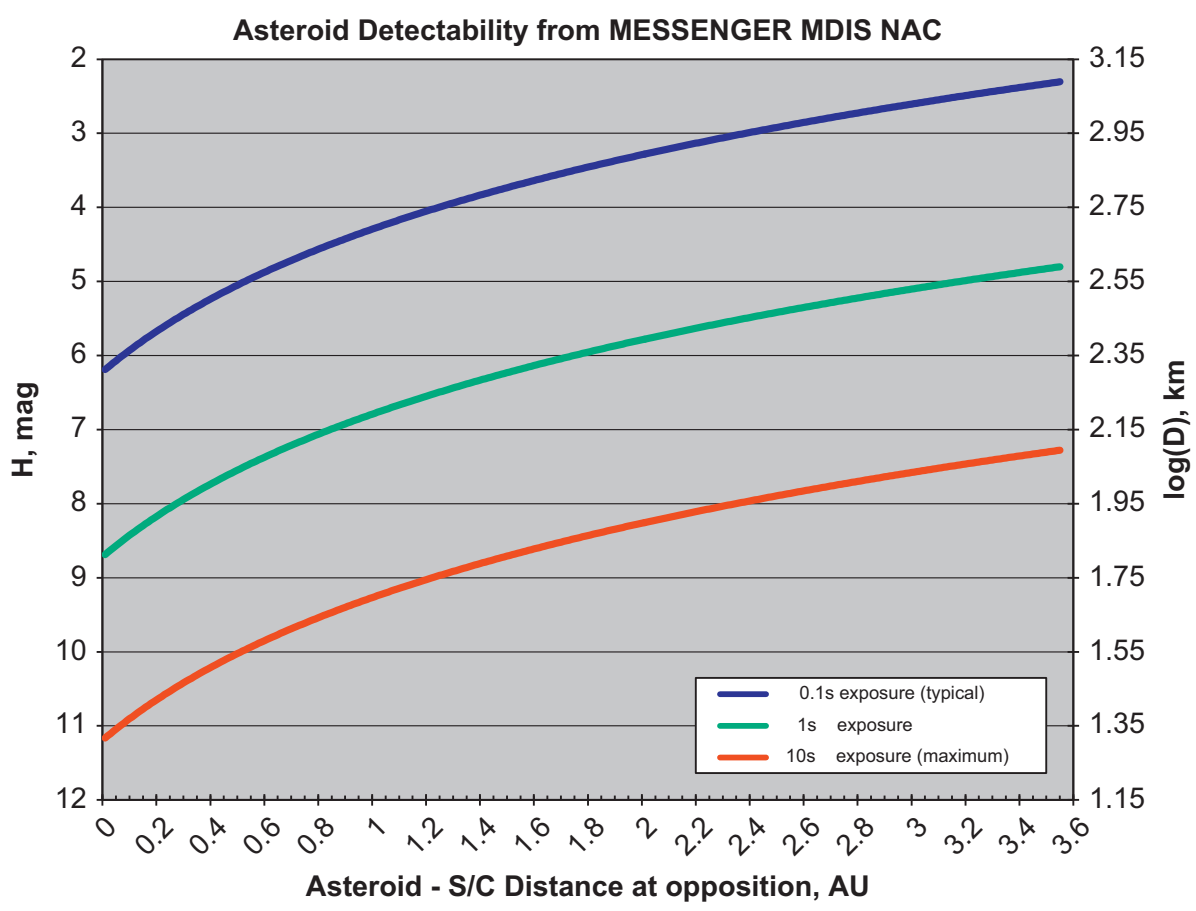

Fig. 2. Detection limit size-distance functions for the MESSENGER MDIS Narrow Angle Camera (NAC).

Zodiacal InterPlanetary Dust (IPD) observations were part of the cruise to Venus

extended Earthguard I-like technology demonstration is feasible

- the long cruise duration can compensate for instrument limitations by offering a larger number of opportunities and wider variation of observation geometry

- limited taxonomy experiments may be included for a subset of the detectable objects
- BeprColombo

- Spectrometers and Imagers for MPO BepiColombo Integrated Observatory System High Resolution Imaging Channel (SIMBIO-SYS HRIC) - Fig. 4

- filter: panchromatic

km-class objects would need to come very close to become detectable

- NEOs of a few $\mathrm{km}$ diameter and larger can be detected out to the near-Earth opposition regions 


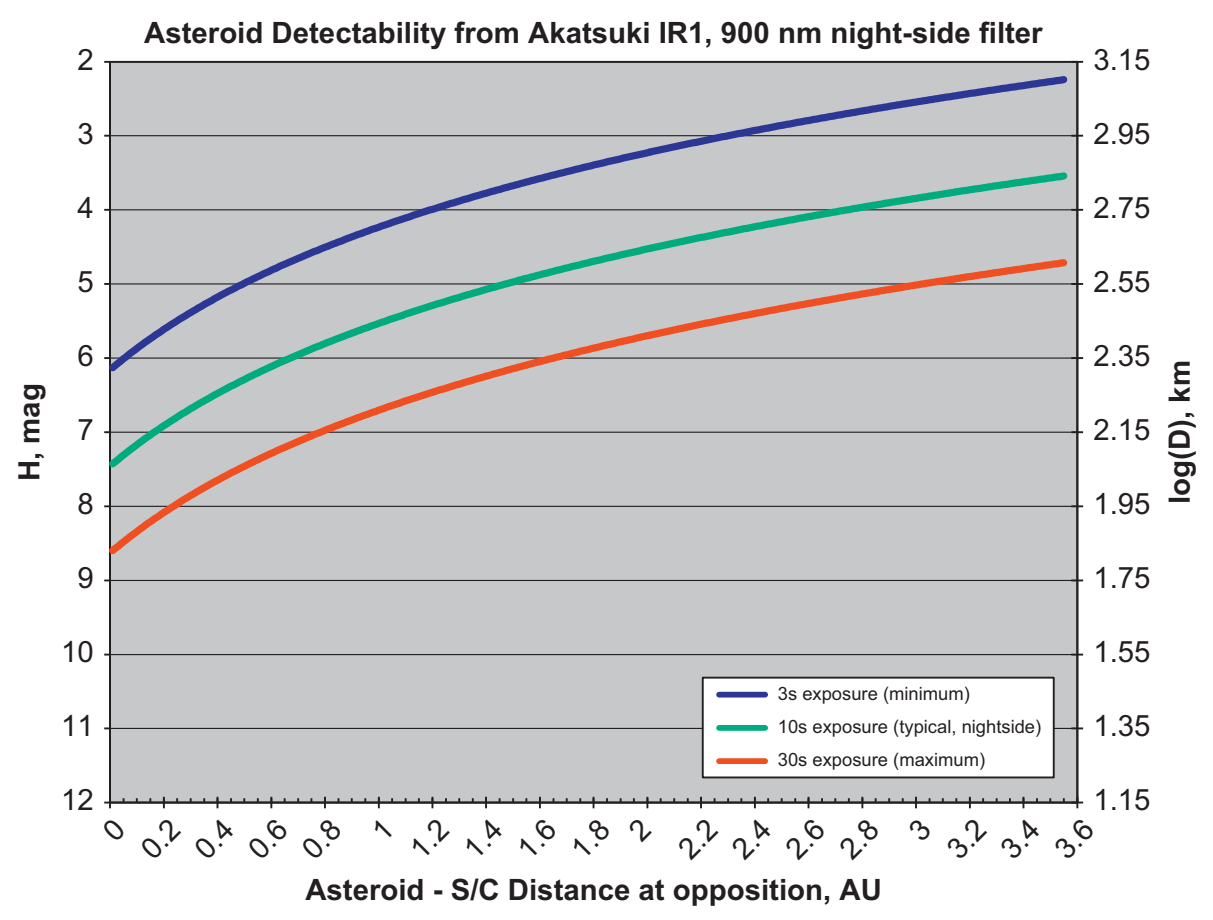

Fig. 3. Detection limit size-distance functions for the AKATsuкI $1 \mu \mathrm{m}$ camera IR1, night-side $900 \mathrm{~nm}$ filter.

- $10 \mathrm{~km}$ class objects can be detected throughout the main belt

- Earthguard I-like observations could be conducted after Mercury orbit insertion,

- survey patterns or region of interest adapted to the small FoV, e.g. investigation of theTrojan regions of Venus and Earth while they are in opposition w.r.t. the spacecraft,

- limited taxonomy potential due to filter bandwidth and imaging channel contrast

\subsubsection{SSSB detection limit size-distance functions for spacecraft cameras}

Figs. 1-4 show the minimum size of detectable objects with respect to the distance from the spacecraft under the conditions given above, for the cameras as previously listed. Note that the three exposure times plotted in each graph vary; where minimum or typical exposure times were not available (cf. Table 1 ) the values have been chosen to represent a reasonable range of the optical design's detection potential. An exposure time of $10 \mathrm{~s}$ is the longest common to all four cameras and therefore shown for comparison of performance. The absolute magnitude, $H$, of a point source target on the left-hand side is converted to a log diameter scale on the right-hand side, using the standard $H$ - $\emptyset$-relationship and assuming a visible albedo of 0.15 . The spacecraft-asteroid distance scale starts at the orbit of Venus and extends along the anti-solar axis. The minimum distance plotted is $0.01 \mathrm{AU}$. Table 2 offers a few notable asteroids as a reference for the $H$ scale and indicates their diameter. [39] Note that the relationship between diameter and absolute magnitude depends of the object's albedo, and that the apparent magnitude at any time depends also on distance, phase angle, viewing geometry, and rotational phase for nonspherical objects.

The Venus Express VMC performance (Fig. 1) clearly shows that it is designed to provide synoptic images of an exceptionally bright area source. It is further restricted by an already fairly narrow filter and minimalist optics, however efficient for its intended purpose.

The MESSENGER MDIS NAC (Fig. 2), also intended to image bright areas, is mainly limited by a short maximum exposure time, and to a lesser extent by its fixed filter's bandwidth. Also, sensitivity for point sources varies depending on the location on the focal plane because the CCD sensor employs anti-blooming gates which reduce active area. [30] With respect to point sources, as an addition to its main task, MDIS was optimised to achieve the requirements of cruise navigation for $\geq 3$ stars per FoV at 7 times read noise, which is mainly carried by the WAC component in the range of 5 to $\sim 8.9$ mag stars but only marginally achieved for NAC at around $8 \mathrm{mag}$. [29] Note that although the sensitivity for the WAC is about 0.6 mag better, its large pixel scale has to be factored in when it comes to the detection of previously unknown objects in front of the variations of the celestial background. A reduced noise margin, e.g. $\mathrm{SNR}=3 \ldots 5$ may be acceptable for point source detection when accurate centroiding is not yet required (e.g. [15]). This would extend the limiting magnitude for either MDIS component by about $1 \mathrm{mag}$. Longer exposure times are not possible due to camera hardware limitations. During cruise, point source observations similar to those proposed herein were undertaken. The limiting magnitude of the MDIS NAC achieved was about 8.5 mag without any special processing. By taking multiple frames and the application of special processing this was extended to about 9.5-10 mag [30]. MESSENGER has by now 


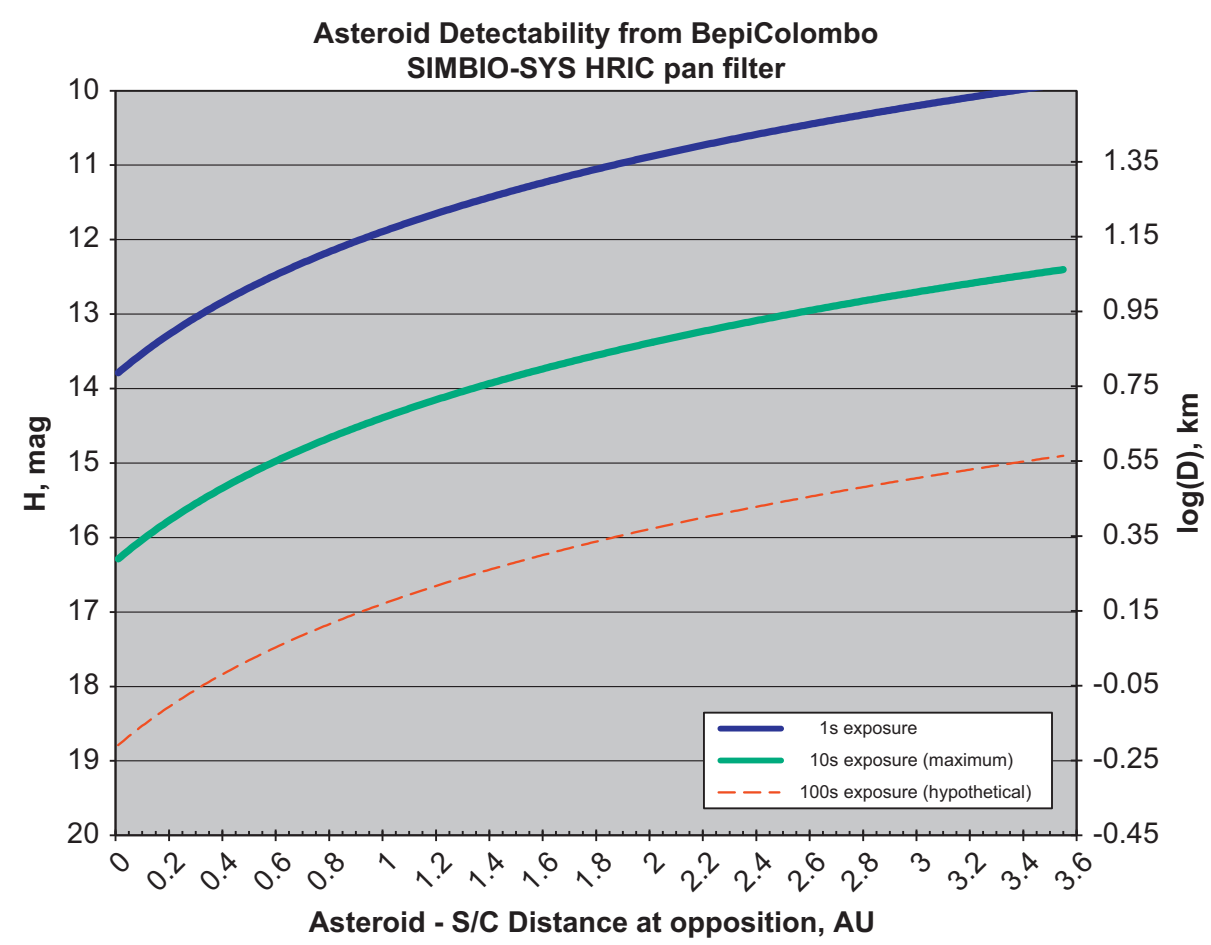

Fig. 4. Detection limit size-distance functions for the BepIColombo SIMBIO-SYS HRIC (panchromatic).

Table 2

Absolute magnitude of notable main belt and near-earth asteroids.

\begin{tabular}{|c|c|c|c|c|c|}
\hline Object & Absolute magnitude $\mathrm{H}$ (mag) & Geometric albedo & Diameter $\varnothing(\mathrm{km})$ & Semi-major axis-a (AU) & Remarks \\
\hline (4) Vesta & 3.20 & 0.4228 & 530 & 2.765 & Brightest main belt asteroid \\
\hline (1) Ceres & 3.34 & 0.0900 & 952 & 2.362 & Largest main belt asteroid \\
\hline (6) Hebe & 5.71 & 0.2679 & 185 & 2.425 & \\
\hline (30) Urania & 7.53 & 0.1714 & 100 & 2.365 & \\
\hline (1036) Ganymed & 9.50 & 0.212 & 38.5 & 2.662 & Largest NEA \\
\hline (433) Eros & 10.31 & 0.24 & 23.3 & 1.458 & First NEA discovered \\
\hline \multirow[t]{2}{*}{ (163693) Atira } & 16.30 & & & 0.741 & Brightest known IEO \\
\hline & 18 & & $\sim 1$ & & First NEA survey goal \\
\hline \multirow[t]{2}{*}{ (99942) Apophis } & 19.7 & 0.33 & 0.27 & 0.922 & Notable PHA \\
\hline & 22 & & $\sim 0.14$ & & Minimum PHA size \\
\hline
\end{tabular}

Using data from [39], objects also selected with respect to classification according to [38].

progressed well into the extended mission phase, and a further extension seems not unlikely. Later mission phases of spacecraft have in the past sometimes opened opportunities for sideline observations.

The AкATsuкI IR2 camera has a stated point source performance of $13 \mathrm{mag}, H$ band, at an exposure time of 120 s. The IR1 camera (Fig. 3) is limited to 30 s exposures. A maximum exposure time capability for the IR1 camera similar to the IR2's would allow it to compensate for the compact optics and narrow filters to an extent almost approaching the MDIS NAC performance for point sources, but at a wider FoV. The IR1 and IR2 cameras are similar and share electronics for $A / D$ conversion since the electronic characteristics of the respective sensors are nearly identical [10]. The pointing accuracy of $0.15^{\circ}(1 \sigma)$ is sufficient for survey-like operations that involve repetitive imaging of the same area, due to the large FoV. Attitude stability at $<0.01^{\circ}$ over $45 \mathrm{~s}$ is better than 1 pixel in the cameras of interest. Since attitude determination accuracy may be slightly worse, reference stars would have to be used, but this is necessary anyway for astrometry. (In the imaging of Venus, limb-fitting is used to improve pointing knowledge) [9]. It may be assumed that the platform also has the ability to support the longexposure performance of the IR2 camera stated in [10].

If similar exposure times were feasible on future large high-resolution cameras such as the BepICoLombo SIMBIO-SYS HRIC (Fig. 4), and could be combined with a (nearly) clear filter mode, a system for the observation of km-scale NEOs and yet smaller IEOs would be available. The limited field of view and high angular resolution of such cameras suggests a useful potential for follow-up observations of objects escaping the reach of ground-based and near-Earth observatories soon after discovery, even if it were not feasible to conduct a more regular population survey during cruise phases of the respective spacecraft. Unfortunately, the BеріCоцомво remote sensing instruments can not operate during cruise because it is blindfolded by the dual spacecraft configuration of MMO and MPO. It can not be used for any observations before these components separate, just before Mercury orbit 
insertion. This would push back any point source observation experiments into the nominal operational science mission phase at the earliest, where such endeavours are unlikely due to data rate constraints, or more likely into later extended mission phases, if any [36]. However, once at Mercury and successfully separated, the BepIColombo SIMBIOSYS HRIC would despite its optimisation for the observation of a bright and hot surface provide the capability to track large NEOs in the vicinity of Earth, and to search for smaller objects in the vicinity of Mercury and Venus.

The proposed NEO survey planning to use the DeEP IMPACT fly-by bus' MRI camera requires a different strategy due to its Earth-like heliocentric orbit. Since it is orbiting within the cloud of NEAs on Earth-like orbits to be observed, and also because the spacecraft design favours such a viewing geometry for thermal and power reasons, a quadrature search is conducted at $\sim 90^{\circ}$ from opposition, focusing on the 'sweet spot' with the highest expected density of such objects. The MRI is the wider angle instrument of the spacecraft but still only has one sixth of the FoV of the BepIColombo HRIC, although with a slightly larger aperture. The survey's main advantage is an observing location distant from Earth, allowing it to place constraints on the population of Earth co-orbitals. [19] A spacecraft in approach to or orbiting Venus or Mercury could search for co-orbitals of these planets in a similar manner. During cruise, especially in low-thrust trajectories, the small-object population across a wide range of heliocentric distances could be sampled by more continuous observations of this kind.

\section{NEO observation feasibility demonstration scenarios}

From the perspective of planetary probe operations, asteroid encounters and observations have long been a welcome bonus objective for the extended periods of interplanetary transfer. After a few initial reservations, the value of such encounters has been recognised as practice targets offering the opportunity to exercise the spacecraft performance envelope, sometimes to carefully selected limits, with the confidence already gained in cruise and calibration operations, on a real object but before any highvalue science results are put at risk. However, these asteroid observations have mostly been close and fast flybys. Observations in the context of Earth-based asteroid surveys and such as intended for EARTHGUARD I require continuous wide-area scanning and regular revisits for the purposes of transient object discovery, motion detection, classification, and tracking. Between these following extremes lies the potential envelope of EARTHGUARD I-like technology demonstrations using existing in-space hardware or future interplanetary spacecraft:

- minimum scenario: one-off observation of a known bright object in opposition to the spacecraft

e.g. Ceres, Vesta, or a large known NEA which happens to be within detection range

- maximum scenario: cruise-phase full asteroid survey implemented aboard the spacecraft

o continuous mosaic imaging; on-board motion detection, processing and autonomy.
First of all, any such a concept needs to be adapted to an existing and carefully optimised spacecraft intended for an entirely different mission. Instruments, thermal design, pointing constraints, and on-board support and services equipment of planetary spacecraft are in many cases designed to a very specific mission scenario. Robust multi-purpose designs tend to be the exception, even at instrument level. Operations during cruise are usually limited to regular health checks or, especially for longer cruise missions, extended software updates to bring the launched hardware into proper operational shape. This approach also addresses concerns that the equipment may in some way wear out by being used extensively before the main target of the mission has been reached. Consequently, a survey experiment will, for example, have to accept the instrument pointing attitude as it results from the spacecraft cruise attitude. The data volume generated may be determined by available onboard storage as well as the housekeeping and telecommand storage and processing requirements aboard. Once arrived at the main mission target, it is unlikely due to the very specific optimisation of planetary spacecraft that sufficient unused capacity exists to support any extended or repetitive additional observations. The capacity of the instruments to generate data usually exceeds the data storage and transmission capabilities, allowing for a changing focus of science interests as well as a rearrangement of the mission concept in the case of an instrument failure.

In keeping with the approach of using presently flying or well advanced and relatively soon available spacecraft designs unmodified, the large number of images usually associated with dedicated survey missions can not be obtained. Also, full on-board motion detection processing is unlikely due to computational capability limits, although some pre-processing such as lossless compression or simple register-stack operations may be possible, if suitable software exists in the instrument. An actual implementation will also depend on the ground-based resources made available. The main bottleneck is the data rate for downlink to Earth at distances of 0.5 to $1.5 \mathrm{AU}$, typically on the order of a few to a few tens of kilobits per second; cf. e.g. [10]. The nominal downlink data rate in X-band for АКАTSUKI varies between 16 and $32 \mathrm{kbps}$ for Earth-spacecraft distances between 1.7 and $0.3 \mathrm{AU}$, respectively. [9] However, an image rate comparable to or even slightly exceeding the respective mission's nominal rate of planetary surface images can be achieved if the mission-typical communication time is provided by the ground network already during cruise. Passage of regions of particular scientific interest in opposition geometry from the spacecraft, e.g. the planet-L4/L5 ecliptic longitudes, may also warrant a temporary higher priority if ground network sharing with other missions is required.

A reasonable higher-end approach for spacecraft with constantly three-axis stabilized cruise attitude may be the use of the spacecraft's most suitable frame camera, with pointing as determined by the design cruise attitude, slowly sweeping out the opposition region of the sky at the angular rate determined by the heliocentric orbit, and with a frame rate set to ensure imaging overlap sufficient for motion 
detection; i.e., most sky-plane locations scanned by the camera FoV due to orbital motion are imaged at least three to four times sequentially. Bulk data rate can be adapted by gaps in coverage in favour of sufficient overlap in the regions still imaged, also in conjunction with downlink sessions if attitude changes are required for transmission. In terms of operations training aspects, such a scenario is probably closer to current planet-orbiter imaging routine than a close and fast asteroid fly-by which nevertheless may well serve to exercise other operational aspects.

A lower-end approach also useful for spacecraft mostly spin-stabilized in cruise may be the extension of regular maintenance phases with temporary tri-axial control, and also the modification of their timing with respect to known favourable asteroid conjunctions, to take a limited set of images of a specific area of the sky. These data can then be downlinked soon, still as part of this operations milestone, or more slowly later, after return to nominal cruise. In a region with less fly-by targets presently known than on trajectories outbound from Earth's orbit, such a limited break from cruise routine may still offer comparable opportunities to exercise ground operations and test at least some spacecraft capabilities on real though distant planetary targets.

Table 3

Spacecraft opposition encounters with selected PHA of interest and Fig. 5 colour code.

\begin{tabular}{lll}
\hline Probe & Asteroid & Closest Approach \\
\hline MESSENGER & Toutatis & $24 / 09 / 2009$ \\
& 1999RQ $_{36}$ & $21 / 09 / 2005$ \\
Aenus Express & Apophis & $28 / 11 / 2006$ \\
Akatsuki & Apophis & $08 / 10 / 2008$ \\
& Apophis & $18 / 08 / 2018$ \\
\hline
\end{tabular}

Using data from [39], objects also selected with respect to classification according to [38].

\subsection{Objects and observation regions of special interest}

From the interior of the respective planet orbits, coorbital objects and L4/5 Trojans become more easily detectable. Also, the structure of the Interplanetary Dust (IPD) may be investigated, including its perturbations caused by the planets. Of particular interest with respect to planetary defence are observations of known PHAs to augment ground-based assets by the different perspective available, or to continuously refine the PHA orbit during periods of invisibility from Earth. As an example, Table 3 and Fig. 5 show selected opposition encounters of three known PHAs with space probes currently in the interior solar system, and their orbits. Note that the orbit of AкATSUKI was propagated from the last available state before the failed Venus orbit insertion, and does not yet include a future Venus orbit insertion. It is therefore known to be inaccurate, but nevertheless believed to represent the general characteristics of the actual current orbit which are similar to the Venus-leading EARTHGUARD I design option using a separate spacecraft. [12] The orbit of Venus is virtually identical to that of Venus Express. BepIColombo is not shown for clarity. The characteristics of its possible envisaged trajectories depend on the actual launch date, but are similar to MESSENGER in general, with the addition of propelled cruise phases. Both present ample geometrical opportunities for observation of regions and objects of special interest in opposition due to the short orbital periods, especially of the revolutions mainly inside of Venus towards Mercury rendezvous.

\subsection{Continuation during the main spacecraft mission}

Once a planetary spacecraft has reached its destination and is safely inserted into orbit around it, some observations may still be conducted when no imaging of value can be obtained from the primary target, e.g. in deep eclipse season when the spacecraft regularly transits the

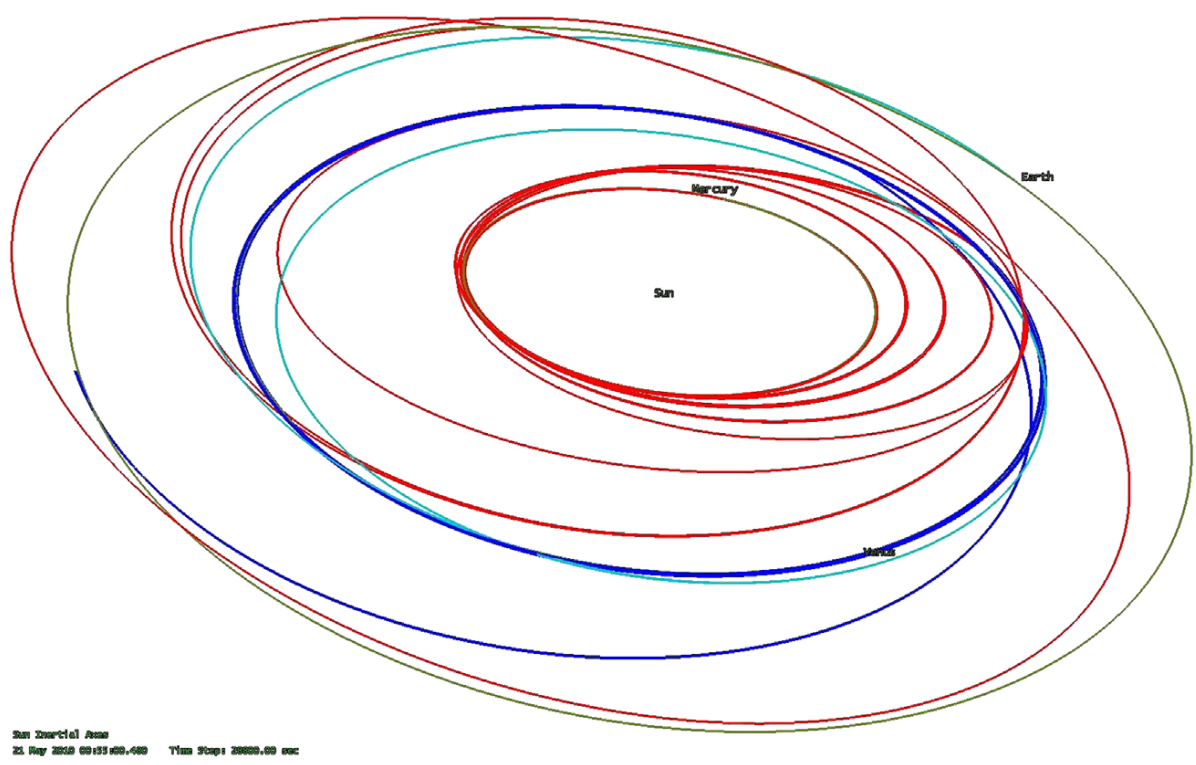

Fig. 5. Orbit geometry of space probes currently in the interior solar system. (MESSENGER - red; Venus Express - light blue; Akatsuki - deep blue.) 
planet's shadow. Then, it may also be possible to use the planet as a sunshield to image parts of the sky usually too close to the Sun, to extend the search for small solar system bodies closer towards the Sun or to continue tracking of objects of special interest there. The latter opportunity is particularly valuable when, with respect to the Earth, the spacecraft with its target planet is in inferior conjunction and the small object of interest close to superior conjunction at elongations too low when seen from Earth.

Finally, the majority of interplanetary spacecraft enters one or more phases of extended missions, sometimes with new science objectives based on the nominal operational phase's results. In other cases, a target object may become exhausted, e.g. because it has been completely mapped and is otherwise free of significant changes, or because the primary mission was a brief one-off event as in the case of DeEp Impact. Then, a significant portion of the spacecraft resources may become available for alternative observations once extended mission phases are entered at good spacecraft health.

\section{Conclusions}

Within certain mission-specific constraints, it is possible to use existing space assets to perform feasibility demonstrations of the observation of small solar system bodies in a mode which is at least on an image level similar to an asteroid survey mission operating in the interior solar system.

Among the cameras studied, the high-resolution cameras on Mercury orbiters have the best detection thresholds, although in a relatively small field of view. If long effective exposure times (on the order of $100 \mathrm{~s}$ in the given designs) can be achieved now by suitable processing of multiple exposures or still implemented in hardware prior to launch, and if sufficient ground-based resources are made available, future cruise operations on the way to Mercury could include pathfinder surveys of the asteroid population interior to Earth's orbit. Such a scenario will include several favourable passes of the planetary Trojan regions in opposition.

The cameras on Venus missions usually have less potential in terms of faint object detection, but provide a wide field of view as well as multiple moderately narrow filter bands ranging from the near ultraviolet to near infrared. During relatively close encounters with larger NEOs, it may be possible to test taxonomy observations; due to the large field of view, this may be possible even if the object's orbit is poorly constrained without taking up too much imaging time. Indeed, at least the IR2 camera on AкATSUKI was designed also with regard to interplanetary dust and faint object observations as a cruise phase objective.

The different viewing geometry is likely to provide opportunities for continued tracking of detectable small objects which have moved into regions of the interior solar system difficult to observe from Earth. This may concern newly discovered objects of general scientific interest as well as PHAs of particular interest to planetary defence.

\section{References}

[1] J.V. McAdams, MESSENGER Mission Overview and Trajectory Design, Astrodynamics Specialist Conference, American Astronautical Society/American Institute of Aeronautics and Astronautics, AAS 03-541, August 3-7, 2003.

[2] J. Fabrega, T. Schirmann, R. Schmidt, D. McCoy, Venus Express on the Right Track, IAC-04-Q.2.a.07, 2003.

[3] M.L. Kaiser, The STEREO mission: an overview, Adv. Space Res. 36 (2005) 1483-1488.

[4] W.D. Pesnell, B.J. Thompson, P.C. Chamberlin, The Solar Dynamics Observatory (SDO), Sol. Phys. 275 (2012) 3-15, http://dx.doi.org/ 10.1007/s11207-011-9841-3.

[5] V. Domingo, B. Fleck, A.I. Poland, The SOHO mission: an overview, Sol. Phys. 162 (1995) 1-37.

[6] C.E. Roberts, The SOHO Mission L1 Halo Orbit Recovery From the Attitude Control Anomalies of 1998, Libration Point Orbits and Applications Conference, 2002.

[7] J. Benkhoff, J. van Casteren, H. Hayakawa, M. Fujimoto, H. Laakso M. Novara, P. Ferri, H.R. Middleton, R. Ziethe, BepiColomboComprehensive exploration of Mercury: Mission overview and science goals, Planet. Space Sci. 58 (2010) 2-20.

[8] T. Imamura, M. Nakamura, M. Ueno, N. Iwagami, T. Satoh, S Watanabe, M. Taguchi, Y. Takahashi, M. Suzuki, T. Abe, G.L Hashimoto, T. Sakanoi, S. Okano, Y. Kasaba, J. Yoshida, M. Yamada N. Ishii, T. Yamada, K. Oyama, PLANET-C: Venus Climate Orbiter mission of Japan, Planetary and Space Science 55 (12) 1831-1842. 10.1016/j.pss.2007.01.009.

[9] M. Nakamura, T. Imamura, N. Ishii, T. Abe, T. Satoh, M. Suzuki, M. Ueno, A. Yamazaki, N. Iwagami, S. Watanabe, M. Taguchi, T. Fukuhara, Y. Takahashi, M. Yamada, N. Hoshino, S. Ohtsuki, K. Uemizu, G.L. Hashimoto, M. Takagi, Y. Matsuda, K. Ogohara N. Sato, Y. Kasaba, T. Kouyama, N. Hirata, R. Nakamura, Y. Yamamoto, N. Okada, T. Horinouchi, M. Yamamoto, Y. Hayashi, Overview of Venus orbiter, Akatsuki, Earth Planets Space 63 (2011) 443-457, http://dx.doi.org/10.5047/eps.2011.02.009.

[10] ISAS, JAXA, Venus Climate Orbiter AKATSUKI / PLANET-C, - 3. Mission Overview, 〈http://www.stp.isas.jaxa.jp/venus/E_plan. html $>4$. Science Instruments and Targets, <http://www.stp.isas.jaxa.jp/venus/E_instrument.html $>$.

[11] JAXA, Venus Climate Orbiter AKATSUKI (PLANET-C)-Topics-AKATSUKI Orbit Control at Perihelion, 〈http://www.jaxa.jp/projects/ sat/planet_c/topics_e.html $\rangle$, updates as of 01 November 2011.

[12] Kayser-Threde, D.L.R., EARTHGUARD I-A Space-Based NEO Detection System-Executive Summary, 2003, 〈http://www.esa.int/gsp/ completed/neo/earthguard1_execsum.pdf $\rangle$.

[13] M. Leipold, A. von Richter, G. Hahn, A.W. Harris (DLR), E. Kührt, H. Michaelis, S. Mottola, "EARTHGUARD I - A NEO Detection Space Mission", Proceedings of Asteroids, Comets, Meteors - ACM 2002 ESA SP-500, http://adsabs.harvard.edu/full/2002ESASP.500.107L.

[14] A.W. Harris (DLR), Man sieht nur die im Dunkeln, die im Lichte sieht man nicht., 〈http://berlinadmin.dlr.de/HofW/nr/138/〉.

[15] R. Findlay, O. Essmann, J.T. Grundmann, H. Hoffmann, E. Kührt, G. Messina, H. Michaelis, S. Mottola, H. Müller, J.F. Pedersen, A SpaceBased Mission to Characterize the IEO Population, IAA Planetary Defence Conference, IAA-WPP-323, Papers, Poster Session, P02_2149803 findlay.pdf, 2011.

[16] A. Hildebrand, B. Gladman, E.F. Tedesco, R.D. Cardinal, P. Gural, M. Granvik, S.M. Larson, K.A. Carroll, P.G. Brown, P. Wiegert, P. Chodas, B.J. Wallace, S.P. Worden, J.M. Matthews, The Near Earth Object Surveillance Satellite (NEOSSat) Will Search near-Sun Along the Ecliptic Plane to Efficiently Discover Objects of the Aten and Atira Orbital Classes, 2011 IAA Planetary Defence Conference, IAA-WPP323, Presentations, Day 1, 1700_Hildebrand May9.pdf.

[17] P.A. Abell, R.G. Mink, J.B. Garvin, B.W. Barbee, D. Mazanek, D.R Komar, D. Adamo, A. Cheng, A.S. Rivkin, K. Hibbard, K.L. Miller, R. Dissly, A. Mainzer, D.K. Yeomans, L.N. Johnson, A Space-Based NearEarth Object Survey Telescope in Support of Human Exploration, Solar System Science, and Planetary Defense, 2011 IAA Planetary Defence Conference, IAA-WPP-323, Presentations, Day 1 , 1725_ABELL_PDC_May-9-2011.pdf.

[18] A. Mainzer, NEOCam The Near-Earth Object Camera, SBAG 2009 November 18

[19] S.R. Chesley, RE: Deep Impact EPOXI NEO search, pers. comm. email 17 August 2012 23:58 UTC

[20] D.L. Hampton, J.W. Baer, M.A. Huisjen, C.C. Varner, A. Delamere, D.D. Wellnitz, M.F. A'Hearn, K.P. Klaasen, An overview of the instrument suite for the DEEP IMPACT mission, Space Sci. Rev. 117 (2005) 43-93, http://dx.doi.org/10.1007/s11214-005-3390-8. 
[21] M.F. A'Hearn, “EPOXI Mission Status Reports”, 〈http://epoxi.umd. edu/1 mission/status.shtml >, 12 July 2012.

[22] D. Beasley, E. Hupp, NASA Announces Deep Impact Future Mission Status, Release: 05-193, 2005.

[23] J.T. Grundmann, W. Halle, S. Montenegro, H. Wüsten, Utilisation of the BIRD Satellite After its End of Operational Life, 4S Symposium 2008, ESA-SP-660, Session_11, Grundmann.pdf.

[24] D.K. Yeomans, A.B. Chamberlin, Comparing the Earth Impact Flux from Comets and Near-Earth Asteroids, IAA Planetary Defence Conference, IAA-WPP-323, Papers, S2, S2_1425_Yeomans.pdf, 2011.

[25] M. Lauer, L. Jauregui, S. Kielbassa, Operational Experience with Autonomous Star Trackers on ESA Interplanetary Spacecraft, 〈http://ntrs.nasa.gov/archive/nasa/casi.ntrs.nasa.gov/ 20080012650_2008012553.pdf $\rangle$.

[26] W.J. Markiewicz et al., VMC: The Venus Monitoring Camera, ESA SP-1295.

[27] G. Piccioni, et al., VIRTIS: The Visible and Infrared Thermal Imaging Spectrometer, ESA SP-1295.

[28] J.L. Bertaux, et al., SPICAV: Spectroscopy for the Investigation of the Characteristics of the Atmosphere of Venus, ESA SP-1295.

[29] S.E. Hawkins III, The Mercury Dual Imaging System on the MESSENGER spacecraft, Space Sci. Rev. 131 (2007) 247-338, http://dx.d oi.org/10.1007/s11214-007-9266-3.

[30] S.E. HawkinsIII, "RE: Exposure Time and Platform Stability of MDIS NAC", pers. comm. email 17AUG2012 16:16 UTC.

[31] W.E. McClintock, M.R. Lankton, The Mercury Atmospheric and Surface Composition Spectrometer for the MESSENGER Mission, Space Sci. Rev. 131 (2007) 481-521, http://dx.doi.org/10.1007/ s11214-007-9264-5.

[32] M. Nakamura, T. Imamura, Present Status of Japanese Venus Climate Orbiter, Fifth Meeting of the Venus Exploration Analysis
Group (VEXAG), May 7-8, 2008-<www.lpi.usra.edu/vexag/ may2008/presentations/3Nakamura.pdf $\rangle$.

[33] N. Iwagami, S. Takagi, S. Ohtsuki, M. Ueno, K. Uemizu, T. Satoh, T. Sakanoi, G.L. Hashimoto, Science requirements and description of the $1 \mu \mathrm{m}$ camera onboard the Akatsuki Venus Orbiter, Earth Planets Space 63 (2011) 487-492, http://dx.doi.org/10.5047/ eps.2011.03.007.

[34] M. Kimata, M. Ueno, H. Yagi, T. Shiraishi, M. Kawai, K. Endo, Y. Kosasayama, T. Sone, T. Ozeki, N. Tsubouchi, PtSi Schottkybarrier infrared focal plane arrays, Opto-Electron. Rev. 6 (1) (1998) $1-10$.

[35] E. Flamini, et al., SIMBIO-SYS: The spectrometer and imagers integrated observatory system for the BepiColombo planetary orbiter, Planet. Space Sci. 58 (2010) (2009) 125-143.

[36] P. Palumbo, Exposure time and platform stability of SIMBIO-SYS HRIC, pers. comm. email 17AUG2012 14:56 UTC.

[37] G.E. Arnold et al., Mercury radiometer and thermal infrared spectrometer-a novel thermal imaging spectrometer for the exploration of Mercury, 2008.

[38] D. Yeomans, R. Baalke, NEO Groups, http://neo.jpl.nasa.gov/neo/ groups.html as of 31 Jan 2011. criteria from other sources or as applied in databases may differ slightly, though not significantly.

[39] for NEOs G. Hahn et al., Table on Physical Properties of NEOs, $\langle$ http://earn.dlr.de/nea/table1_new.html updated 20JUL2012〉 and A. Milani et al., NEODyS-2 Near Earth Objects-Dynamic Site, 〈http://newton.dm.unipi.it/neodys/index.php?pc $=0$ as of 23AUG2012 $\rangle$; for main belt objects JPL Small-Body Database Browser, 〈http://ssd.jpl.nasa.gov/sbdb.cgi as of 23AUG2012 〉. Data for objects listed in both sources, from other sources, or as applied in databases may differ slightly, though not significantly. 